\title{
Preferences for Gender Affirming Treatment and Associated Factors Among Transgender People in Sweden
}

\author{
Cathrine Axfors ${ }^{1}$ - Stavros I. Iliadis ${ }^{2}$ (D) Lovisa L. Rasmusson ${ }^{1}$ - Ulrika Beckman ${ }^{3}$. Attila Fazekas ${ }^{4} \cdot$ Louise Frisén $^{5}$. \\ Lotta Sandström $^{6} \cdot$ Nils Thelin $^{7} \cdot$ Jeanette Wahlberg ${ }^{8} \cdot$ Alkistis Skalkidou $^{2} \cdot$ Fotios C. Papadopoulos $^{1}$
}

Accepted: 23 September 2021

(c) The Author(s) 2022

\begin{abstract}
Introduction Gender affirming surgery of primary and/or secondary sex characteristics has been shown to alleviate gender dysphoria. A descriptive snapshot of current treatment preferences is useful to understand the needs of the transgender population seeking health care. This study aimed to describe preferences for gender affirming treatment, and their correlates, among individuals seeking health care for gender dysphoria in Sweden after major national legislative reforms.

Methods Cross-sectional study where transgender patients $(n=232)$ recruited from all six Gender Dysphoria centers in Sweden 2016-2019, answered a survey on treatment preferences and sociodemographic, health, and gender identity-related information during the same time-period. Factors associated with preferring top surgery (breast augmentation or mastectomy), genital surgery, and other surgery (e.g., facial surgery) were examined in univariable and multivariable regression analyses in the 197 people without prior such treatment. Main study outcomes were preferences for feminizing or masculinizing hormonal and surgical gender affirming treatment.

Results The proportion among birth assigned male and assigned female patients preferring top surgery was $55.6 \%$ and $88.7 \%$, genital surgery $88.9 \%$ and $65.7 \%$, and other surgery (e.g., facial surgery) $85.6 \%$ and $22.5 \%$, respectively. Almost all participants (99.1\%) wanted or had already received hormonal treatment and most (96.7\%) wished for some kind of surgical treatment; 55.0\% wanted both top and genital surgery. Preferring a binary pronoun (he/she) and factors indicating more severe gender incongruence were associated with a greater wish for surgical treatment. Participants with somatic comorbidities were less likely to want genital surgery, while aF with lacking social support were less likely to want internal genital surgery, in the multivariable analyses.

Conclusions In this sample of Swedish young adults seeking health care for gender dysphoria, preferences for treatment options varied according to perceived gender identity.

Policy Implications The study findings underline the need for individualized care and flexible gender affirming treatment options. The role of somatic comorbidities should be further explored, and support should be offered to transgender people in need. There is an unmet need for facial surgery among aM.
\end{abstract}

Keywords Gender confirmation procedures · Gender dysphoria · Gender identity · Health services for transgender persons · Transgender persons

Stavros I. Iliadis

stavros.iliadis@kbh.uu.se

1 Department of Neuroscience, Uppsala University, 75185 Psychiatry, Sweden

2 Department of Women's and Children's Health, Uppsala University, 75185 Uppsala, Sweden

3 Department of Psychiatry, Södra Älvsborgs Hospital, 44130 Alingsås, Sweden

4 Department of Psychiatry, Lund University, 28521 Lund, Sweden
5 Department of Clinical Neuroscience, Karolinska Institutet, 17177 Stockholm, Sweden

6 Department of Clinical Sciences, Umeå University, 90187 Umeå, Sweden

7 Division of Psychiatry, Linköping University Hospital, 58185 Linköping, Sweden

8 Department of Endocrinology and Department of Health, Medicine and Caring Sciences, Linköping University, 58183 Linköping, Sweden 


\section{Introduction}

Transgenderism is an umbrella term for gender identities that differ from one's birth assigned sex, according to gender roles dictated by prevailing cultural norms. The term admits a gender spectrum (Richards et al., 2016). The diagnosis Gender Dysphoria refers to the strong and persistent distress one may experience as a result from the incongruence between one's gender identity and birth assigned sex (American Psychiatric Association, 2013). Minority stress, discrimination, abuse, ignorance, and prejudice from society at large as well as within families contribute to transgender persons' risk of mental suffering (Winter et al., 2016), and psychiatric morbidity is common (Claes et al., 2015; Dhejne et al., 2016; Heylens et al., 2014). Quality of life for people with gender dysphoria may be improved through gender affirming treatment, seeking to better align physical appearance with gender identity.

According to a systematic review, gender affirming hormonal therapy improved quality of life and sexual function as well as decreased gender dysphoria and psychiatric morbidity for $72-80 \%$ of the patients (Murad et al., 2010). Gender affirming surgery of primary and/or secondary sex characteristics has been shown to alleviate gender dysphoria, raise the chances of being perceived according to gender identity, and improve quality of life (van de Grift et al., 2018) as well as global functioning (Johansson et al., 2010), although studies generally have low response rates and few participants. In Swedish treatment guidelines for so-called "top surgery" (The National Board of Health \& Welfare, 2015), breast augmentation is a possibility for aM individuals after a year of potential hormonal treatment, and $\mathrm{aF}$ individuals may request mastectomy or masculinizing chest surgery. Feminizing genital surgery may be comprised of removal of the penis and/or testes together with the construction of a neovagina, clitoris and labiae (Coleman et al., 2012). Masculinizing genital surgery can be external (phalloplasty or metoidioplasty) and/or internal (hysterectomy and salpingo-oophorectomy). Genital surgery complications related to tissue damage and scarring are more common in masculinizing than feminizing genital surgery, with more complications for phalloplasty than metoidioplasty (Pan \& Honig, 2018). Hormonal treatment for aM individuals does not alter facial bone structures, thus feminizing facial surgery may be a preference, as well as thyroid cartilage reduction (chondrolaryngoplasty) (Coleman et al., 2012). For aF individuals, examples of available procedures additional to genital and top surgery are hip liposuction or pectoral implants. Other gender affirming treatments may additionally involve speech therapy or hair removal by laser or electrolysis (The National Board of Health \& Welfare, 2015).
There are two main reasons why it is timely to investigate contemporary treatment preferences in people seeking healthcare for gender dysphoria. First, while previously having a binary gender identity and stating a wish for all available treatment was often expected in order to receive any treatment for gender dysphoria (Cohen-Kettenis \& Pfäfflin, 2010), currently the gender spectrum paradigm has opened up for more individualized treatment. Second, the Swedish setting has seen major legislative reform, discarding the prerequisites of sterilization and unmarried civil status for gender affirmative genital surgery that were in effect until 2013 (Ministry of Social Affairs, 1972); however, treatment preferences have not been investigated since. In 1960-2010, 89\% of transgender people applying for legal sex reassignment in Sweden underwent genital surgery (Dhejne et al., 2014). Applications for legal sex reassignment have markedly increased per 100,000 inhabitants and year, being 0.80 in 2010, 1.76 in 2013, and 4.43 in 2018, and in absolute numbers 75,170 , and 453 per year, respectively (Statistics Sweden (SCB), 2019; The National Board of Health \& Welfare, 2019). A descriptive snapshot of treatment preferences is useful to understand the needs of the transgender population seeking health care.

\section{Aims of the Study}

The overall aim was to describe the preferences for gender affirming treatment, and their correlates among sociodemographic and other health-related information in addition to gender identity characteristics, in individuals seeking health care for gender dysphoria in Sweden.

\section{Materials and Methods}

\section{Procedure}

This project is part of the Swedish Gender Dysphoria Study (Svenska Könsdysforistudien, SKDS), an ongoing longitudinal study at the six regional centers responsible for the evaluation process of gender dysphoria in Sweden (Alingsås/ Gothenburg, Linköping, Lund/Malmö, Stockholm, Umeå, and Uppsala), most of which accept self-referrals. The SKDS consists of several web-based surveys and self-report inventories to be answered at 13 different occasions distributed over 3 years. This cross-sectional sub-study comprises data from the initial survey at study inclusion and a psychometric instrument measuring gender dysphoria at the second week after inclusion. The survey was designed by one of the authors (FCP) in collaboration with colleagues from the multidisciplinary gender dysphoria team at Uppsala University Hospital. 


\section{Participants}

Between late 2016 and June 2019, individuals aged 15 years or more, who were initiating or already had an established health care contact in psychiatry, gynecology, or endocrinology at any of the six gender dysphoria centers in Sweden, were asked to participate in the SKDS. The participants were required to have basic knowledge in Swedish. In this study, participants were excluded if they had incomplete submission of basic demographic data. In the association analyses, we furthermore excluded those who had already had gender affirming surgery, since some potential predictors for treatment preferences (e.g., satisfaction with body appearance) may change after treatment.

\section{Study Variables}

For a complete description of the coding of survey responses, see Supplement 1. Outcome variables were genital surgery (referring to external genital surgery), internal genital surgery, hormonal treatment, top surgery (breast augmentation for aM individuals, mastectomy for aF individuals), and other surgery (e.g., facial surgery, or for aM individuals chondrolaryngoplasty or vocal cord surgery). Sociodemographic information included age, partner status, educational level, work status, and perceived social support regarding gender dysphoria. Regarding health, participants reported their body mass index $\left(\mathrm{BMI}, \mathrm{kg} / \mathrm{m}^{2}\right)$, somatic and psychiatric morbidity, any previous suicidal attempts, suicidal thoughts or self-harm during the past year, and perceived health (continuous 100-point scale from worst to best imaginable).

Information related to gender identity and expression included debut age of experiencing gender incongruence, preferred pronoun (with binary indicated by he/she, and non-binary indicated by "other" or "hen" in Swedish, a gender-neutral pronoun corresponding to the English singular "they"), identification with birth assigned gender and with opposite to birth assigned gender (continuous 100-point scale from not at all to entirely), and satisfaction with the body appearance (continuous 100-point scale from very displeased to very pleased). Respondents were also asked whether currently living outwardly according to their gender identity, whether being sexually active, about their sexual attraction patterns, if they had experienced discrimination because of their gender identity or expression during the past year (continuous 100-point scale from none at all to a lot), and if they had ever experienced trauma (e.g., threats, physical violence, or non-consensual sexual contact) because of their gender identity or expression.

To assess gender dysphoria, we used the Swedish translation of the Transgender Congruence Scale (TCS) (Södersten, 2016), a 12-item, 5-point Likert scale questionnaire regarding a person's experience of gender identity during the latest 2 weeks. The TCS is believed to be more inclusive for fluctuating and non-binary gender identities than other scales (Kozee et al., 2012) and has two subscales for which mean scores are calculated: Appearance Congruence (items 1-9) and Gender Identity Acceptance (items 10-12). Lower mean scores indicate a higher grade of gender dysphoria. In this sample, the Appearance Congruence and Gender Identity Acceptance subscales had Cronbach's alpha of 0.93 and 0.78 , respectively.

\section{Statistical Analyses}

Analyses were performed with R version 3.5.3 (2019-0311 ), and statistical significance was set at two-tailed $p<0.05$. We present descriptive statistics for the total sample as well as divided by assigned sex ( $\mathrm{aM}$ and $\mathrm{aF}$ ) regarding preferences for gender affirming treatment and background information. Univariable associations between preferences for gender affirming treatment and background information variables were examined separately by assigned sex, using Pearson's chi-square tests for categorical variables and Mann-Whitney $U$ test for scale variables. Hormonal treatment was not included in the association analyses since almost the entire sample expressed that preference (99.1\%).

Multivariable logistic regression analyses were used to identify variables that were independently associated with preferences for gender affirming treatment. The dependent variables were defined as top surgery, genital surgery, internal genital surgery, and other surgery. Except for internal genital surgery, only applicable to the aF group, analyses were run in the total sample. As predictor variables, we added those variables that were associated with the outcomes in the univariable analyses at $p<0.10$ in either of the $\mathrm{aM}$ or $\mathrm{aF}$ groups. If both identification with birth assigned gender and identification with opposite to birth assigned gender were identified as relevant, we chose only the first because of collinearity. Additionally, we considered assigned sex, age, social support, binary pronoun, and our indicator of gender dysphoria (the TCS subscale Appearance Congruence) as theoretically relevant covariables and added these for all outcomes except internal genital surgery because of the smaller sample size.

\section{Results}

\section{Descriptive Statistics}

A flowchart of the participants is shown in Fig. 1. Most of the participants were recruited in central and southern Sweden (Uppsala, Linköping and Lund). The final sample comprised $90(38.8 \%) \mathrm{aM}$ and $142(61.2 \%) \mathrm{aF}$ individuals. 


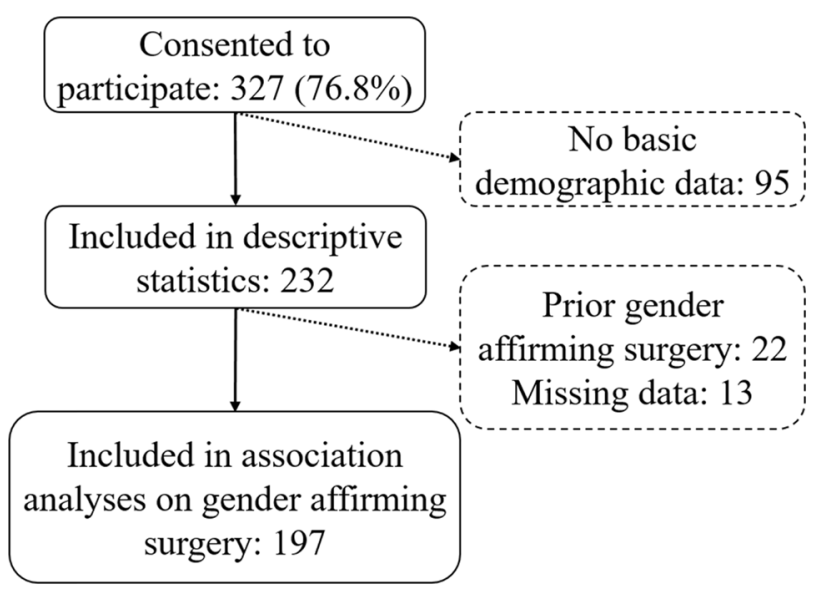

Fig. 1 Flowchart of participants. Exclusions in boxes with dotted lines

On average, participants filled out the survey two years after their first contact with a gender dysphoria center (median 1.2 years, interquartile range $(\mathrm{IQR})=1.9$ years, range 1.3 weeks to 24 years). Half of the sample reported having received a diagnosis of Gender Dysphoria or Transsexualism, while the other half still participated in assessments and had not received any diagnosis. Excluded from the association analyses were 22 participants that had received prior gender affirming surgery (breast augmentation $(n=1)$, feminizing genital surgery $(n=7)$, feminizing facial surgery $(n=1)$, chondrolaryngoplasty $(n=3)$, mastectomy $(n=9)$, and/or masculinizing genital surgery $(n=3)$ ).

In this sample, almost everyone wanted or had already started hormonal gender affirming treatment $(n=230$, $99.1 \%)$. Similarly, almost everyone wanted or had already received some form of surgical gender affirming treatment ( $n=223,96.7 \%$, missing $n=2$ ). Those wishing for or having already received "full" surgical treatment (top surgery and genital surgery) in the total sample, among aM, and among $\mathrm{aF}$, were 127 (55.0\%), 47 (52.2\%), and 80 (56.7\%), respectively. For aM individuals, slightly more than half wanted top surgery, $80(88.9 \%)$ wanted or had already had genital surgery, and $77(85.6 \%)$ expressed a wish for some sort of other surgery. For aF individuals, $126(88.7 \%)$ wanted top surgery, $92(64.8 \%)$ wanted or had already had (external) genital surgery, $92(64.8 \%$ ) wanted internal genital surgerym and $32(22.5 \%)$ opted for some sort of other surgery. Other descriptive results concerning treatment preferences are shown in Table 1.

Background information is presented in Table 2. Participants were commonly in their late twenties, not in a relationship, and the majority had more than 12 years of schooling. Seventy-five (33.6\%) participants had a BMI > 27, exceeding the maximum limit for genital surgery in Sweden (aM, $n=26,30.2 \%$; aF, $n=49,35.8 \%$ ). Most people identified with a binary pronoun $(n=206,88.8 \%)$, identified strongly
Table 1 Preferences for gender affirming treatment in the total sample and divided by assigned male (aM) and assigned female (aF) sex at birth

\begin{tabular}{lllll}
\hline Treatment & Preference & aM, $\boldsymbol{n = 9 0}$ & $\mathbf{a F , ~} \boldsymbol{n = 1 4 2}$ & Total, $\boldsymbol{n = 2 3 2}$ \\
\hline Hormonal & Absolutely & $65(72.2)$ & $94(66.2)$ & $159(68.5)$ \\
& To some extent & $1(1.1)$ & $11(7.7)$ & $12(5.2)$ \\
& Not really & $0(0)$ & $0(0)$ & $0(0)$ \\
& Not at all & $1(1.1)$ & $1(0.7)$ & $2(0.9)$ \\
& Having/have already had & $22(24.4)$ & $35(24.6)$ & $57(24.6)$ \\
Top surgery & Missing & $1(1.1)$ & $1(0.7)$ & $2(0.9)$ \\
Genital surgery & Yes & $50(55.6)$ & $126(88.7)$ & $176(75.9)$ \\
& No & $40(44.4)$ & $16(11.3)$ & $56(24.1)$ \\
& Absolutely & $52(57.8)$ & $37(26.1)$ & $89(38.4)$ \\
To some extent & $21(23.3)$ & $52(36.6)$ & $73(31.5)$ \\
& Not really & $6(6.7)$ & $33(23.2)$ & $39(16.8)$ \\
& Not at all & $3(3.3)$ & $15(10.6)$ & $18(7.8)$ \\
& Having/have already had & $7(7.8)$ & $3(2.1)$ & $10(4.3)$ \\
Othernal genital surgery surgery & Missing & $1(1.1)$ & $2(1.4)$ & $3(1.3)$ \\
& Hysterectomy (Yes) & - & $82(57.7)$ & - \\
& Salpingo-oophorectomy (Yes) & - & $88(62)$ & - \\
& Facial (Yes) & $62(68.9)$ & $17(12)$ & $79(34.1)$ \\
& Chondrolaryngeal (Yes) & $50(55.6)$ & - & - \\
& Vocal cord (Yes) & $42(46.7)$ & - & - \\
& Other surgery (Yes) & $25(27.8)$ & $20(14.1)$ & $45(19.4)$ \\
\hline
\end{tabular}

All cells show $n(\%)$

${ }^{\mathrm{a}}$ Breast augmentation $(\mathrm{aM})$ or mastectomy $(\mathrm{aF})$ 
Table 2 Background information of the total sample and divided by assigned male $(\mathrm{aM})$ and assigned female (aF) sex at birth

\begin{tabular}{|c|c|c|c|c|}
\hline \multicolumn{2}{|l|}{ Variable } & \multirow{2}{*}{$\begin{array}{l}\mathbf{a M}, \boldsymbol{n}=\mathbf{9 0} \\
27.0(14.0)\end{array}$} & \multirow{2}{*}{$\begin{array}{l}\mathbf{a F}, \boldsymbol{n}=\mathbf{1 4 2} \\
23.0(8.0)\end{array}$} & \multirow{2}{*}{$\begin{array}{l}\text { Total, } \boldsymbol{n}=\mathbf{2 3 2} \\
24.0(10.0)\end{array}$} \\
\hline Age & Median (IQR) & & & \\
\hline \multirow[t]{2}{*}{ Partner status $^{\mathrm{a}}$} & In a relationship & $24(26.7)$ & $43(30.3)$ & $67(28.9)$ \\
\hline & Single & $64(71.1)$ & $88(62)$ & $152(65.5)$ \\
\hline \multirow[t]{2}{*}{ Educational level } & $\leq 12$ years & $49(54.4)$ & $99(69.7)$ & $148(63.8)$ \\
\hline & $>12$ years & $41(45.6)$ & $43(30.3)$ & $84(36.2)$ \\
\hline \multirow[t]{3}{*}{ Work status $^{\mathrm{a}}$} & Working & $38(42.2)$ & $47(33.1)$ & $85(36.6)$ \\
\hline & Unemployed/sick-leave & $31(34.4)$ & $45(31.7)$ & $76(32.8)$ \\
\hline & Other & $16(17.8)$ & $43(30.3)$ & $59(25.4)$ \\
\hline \multirow[t]{3}{*}{ Perceived social support $^{\mathrm{b}}$} & Good & $38(42.2)$ & $74(52.1)$ & $112(48.3)$ \\
\hline & Partially/none & $49(54.4)$ & $63(44.4)$ & $112(48.3)$ \\
\hline & I have no gender dysphoria anymore & $3(3.3)$ & $5(3.5)$ & $8(3.4)$ \\
\hline Body mass index & Median (IQR) & $24.6(6.5)$ & $24.0(7.7)$ & $24.2(7.3)$ \\
\hline \multirow[t]{2}{*}{ Somatic morbidity } & Yes & $44(48.9)$ & $57(40.1)$ & $101(43.5)$ \\
\hline & No & $46(51.1)$ & $85(59.9)$ & $131(56.5)$ \\
\hline \multirow[t]{2}{*}{ Psychiatric morbidity } & Yes & $55(61.1)$ & $105(73.9)$ & $160(69)$ \\
\hline & No & $35(38.9)$ & $37(26.1)$ & $72(31)$ \\
\hline \multirow[t]{2}{*}{ Any suicidal attempts ${ }^{\mathrm{a}}$} & Yes & $19(21.1)$ & $47(33.1)$ & $66(28.4)$ \\
\hline & No & $70(77.8)$ & $94(66.2)$ & $164(70.7)$ \\
\hline \multirow[t]{2}{*}{ Suicidal thoughts or self-harm past year ${ }^{a}$} & Yes & $39(43.3)$ & $65(45.8)$ & $104(44.8)$ \\
\hline & No & $49(54.4)$ & $76(53.5)$ & $125(53.9)$ \\
\hline Perceived health & $\begin{array}{l}\text { Continuous scale from worst imaginable }=1 \text { to best } \\
\text { imaginable }=100 . \text { Median }(\mathrm{IQR})\end{array}$ & $60.0(45.8)$ & $50.0(35.8)$ & $55.0(40.2)$ \\
\hline Debut age of gender incongruence & Median (IQR) & $13.0(6.8)$ & $12.5(8.0)$ & $13.0(7.2)$ \\
\hline \multirow[t]{4}{*}{ Preferred pronoun } & $\mathrm{He}$ & $4(4.4)$ & $129(90.8)$ & $133(57.3)$ \\
\hline & She & $71(78.9)$ & $2(1.4)$ & $73(31.5)$ \\
\hline & They ("Hen" in Swedish) & $13(14.4)$ & $11(7.7)$ & $24(10.3)$ \\
\hline & Other & $2(2.2)$ & $0(0)$ & $2(0.9)$ \\
\hline Identification with birth assigned sex & $\begin{array}{l}\text { Continuous scale from not at all=1 to entirely }=100 \text {. } \\
\text { Median (IQR) }\end{array}$ & $10.0(21.0)$ & $1.0(7.0)$ & $3.0(13.0)$ \\
\hline Identification with opposite to birth assigned sex & $\begin{array}{l}\text { Continuous scale from not at all = } 1 \text { to entirely }=100 \text {. } \\
\text { Median (IQR) }\end{array}$ & $86.5(20.0)$ & $94.0(18.0)$ & $90.0((20.0)$ \\
\hline \multirow[t]{4}{*}{ Real-life experience $^{a}$} & Always & $37(41.1)$ & $105(73.9)$ & $142(61.2)$ \\
\hline & Often & $16(17.8)$ & $24(16.9)$ & $40(17.2)$ \\
\hline & Sometimes & $26(28.9)$ & $11(7.7)$ & $37(15.9)$ \\
\hline & Never & $10(11.1)$ & $2(1.4)$ & $12(5.2)$ \\
\hline Satisfaction with body appearance & $\begin{array}{l}\text { Continuous scale from very displeased }=1 \text { to very } \\
\text { pleased }=100 . \text { Median }(\mathrm{IQR})\end{array}$ & $20.0(23.5)$ & $13.0(26.0)$ & $16.5(27.0)$ \\
\hline \multirow[t]{5}{*}{ Sexual attraction pattern ${ }^{a}$} & Androphilic & $18(20)$ & $35(24.6)$ & $53(22.8)$ \\
\hline & Gynophilic & $45(50)$ & $47(33.1)$ & $92(39.7)$ \\
\hline & Bisexual & $16(17.8)$ & $17(12)$ & $33(14.2)$ \\
\hline & Asexual & $5(5.6)$ & $19(13.4)$ & $24(10.3)$ \\
\hline & Unsure/Other & $5(5.6)$ & $24(16.9)$ & $29(12.5)$ \\
\hline \multirow[t]{2}{*}{ Sexually active ${ }^{a}$} & Yes & $44(48.9)$ & $69(48.6)$ & $113(48.7)$ \\
\hline & No & $46(51.1)$ & $71(50)$ & $117(50.4)$ \\
\hline $\begin{array}{l}\text { Discrimination during past year because of gender } \\
\text { identity and expression }\end{array}$ & $\begin{array}{l}\text { Continuous scale from no discrimination at all }=1 \text { to a } \\
\text { lot of discrimination }=100 . \text { Median }(I Q R)\end{array}$ & $19.5(54.2)$ & $36.0(52.0)$ & $29.0(53.5)$ \\
\hline \multirow{2}{*}{$\begin{array}{l}\text { Experienced lifetime trauma because of gender } \\
\text { identity and expression }\end{array}$} & Yes & $41(45.6)$ & $63(44.4)$ & $104(44.8)$ \\
\hline & No & $47(52.2)$ & $77(54.2)$ & $124(53.4)$ \\
\hline TCS Appearance Congruence subscale & Median (IQR) & $1.9(1.3)$ & $1.8(1.0)$ & $1.8(1.2)$ \\
\hline TCS Gender Identity Acceptance subscale & Median (IQR) & $4.0(1.7)$ & $4.0(1.1)$ & $4.0(1.3)$ \\
\hline
\end{tabular}

Cells show $n(\%)$ if not otherwise stated

$I Q R$ interquartile range, TCS Transgender Congruence Scale

${ }^{a}$ Counts and percentages do not sum because of missing data

${ }^{\mathrm{b}}$ Perceived social support regarding gender dysphoria 
with the binary opposite to their birth assigned sex, and lived openly according to their gender identity $(n=182,78.8 \%)$. Almost every second participant reported lifetime experience of trauma related to their gender identity and expression, weak or no social support, and suicidal thoughts or self-harm without suicidal intention during the past year. More than one in four participants reported suicidal attempts during their life.

\section{Univariable Associations}

For aM individuals without previous gender-affirming surgery (Table 3), those who wanted genital surgery more often had previous suicidal ideation or self-harm, chose a binary pronoun (she), identified themselves to a higher extent with a female gender, and were less often sexually active than those who did not want genital surgery. The only factor associated with a wish for other surgery (facial, chondrolaryngoplasty, vocal cord, or other) was a stronger identification with a male gender.

For aF individuals without previous gender-affirming surgery (Table 4), people who wanted top surgery identified to a lower degree with a female gender, and reported a lower satisfaction with their body appearance and more severe gender incongruence than those who did not want top surgery. Expressing a wish for (external) genital surgery was associated with preferring a binary pronoun (he), identifying to a lower extent with a female gender and to a higher extent with a male gender, and reporting a gynophilic sexual attraction pattern. Several factors were

Table 3 Preferences for gender affirming treatments and associated factors for participants assigned male sex at birth

\begin{tabular}{|c|c|c|c|c|c|c|}
\hline \multirow[t]{2}{*}{ Assigned male } & \multicolumn{2}{|c|}{$\begin{array}{l}\text { Top surgery (breast } \\
\text { augmentation) }\end{array}$} & \multicolumn{2}{|c|}{ Genital surgery } & \multicolumn{2}{|c|}{$\begin{array}{l}\text { Other surgery (e.g., } \\
\text { facial) }\end{array}$} \\
\hline & No, $n=32$ & Yes, $n=42$ & No, $n=6$ & Yes, $n=67$ & No, $n=8$ & Yes, $n=66$ \\
\hline Age, median (IQR) & $24.0(9.0)$ & $27.0(15.0)$ & $30.0(18.0)$ & $25.0(11.0)$ & $21.0(9.5)$ & $26.0(12.2)$ \\
\hline Partner status (single) & $23(71.9 \%)$ & $29(69 \%)$ & $2(33.3 \%)$ & $49(73.1 \%)$ & $4(50 \%)$ & $48(72.7 \%)$ \\
\hline Educational level ( $\leq 12$ years) & $15(46.9 \%)$ & $27(64.3 \%)$ & $2(33.3 \%)$ & $39(58.2 \%)$ & $6(75 \%)$ & $36(54.5 \%)$ \\
\hline Working (not unemployed/sick-leave/other) & $11(34.4 \%)$ & $18(42.9 \%)$ & $3(50 \%)$ & $26(38.8 \%)$ & $1(12.5 \%)$ & $28(42.4 \%)$ \\
\hline Good social support & $10(31.2 \%)$ & $22(52.4 \%)$ & $2(33.3 \%)$ & $30(44.8 \%)$ & $4(50 \%)$ & $28(42.4 \%)$ \\
\hline Body mass index, median (IQR) & $23.8(5.6)$ & $24.7(6.2)$ & $24.6(3.4)$ & $24.4(6.8)$ & $25.4(8.6)$ & $24.1(6.3)$ \\
\hline Somatic morbidity & $14(43.8 \%)$ & $20(47.6 \%)$ & $4(66.7 \%)$ & $29(43.3 \%)$ & $3(37.5 \%)$ & $31(47 \%)$ \\
\hline Psychiatric morbidity & $19(59.4 \%)$ & $26(61.9 \%)$ & $3(50 \%)$ & $41(61.2 \%)$ & $5(62.5 \%)$ & $40(60.6 \%)$ \\
\hline $\begin{array}{l}\text { Any suicidal attempts, or past year suicidal ideation or self- } \\
\text { harm }\end{array}$ & $15(46.9 \%)$ & $24(57.1 \%)$ & $\mathbf{0}(\mathbf{0} \%)$ & $39(58.2 \%)$ & $2(25 \%)$ & $37(56.1 \%)$ \\
\hline Perceived health, median (IQR) & $62.0(42.0)$ & $54.0(47.0)$ & $31.0(35.0)$ & $61.0(44.2)$ & $70.0(38.0)$ & $60.0(46.5)$ \\
\hline Debut age of gender incongruence & $14.0(3.8)$ & $12.0(8.0)$ & $14.5(20.5)$ & $13.0(6.5)$ & $14.5(20.5)$ & $13.0(6.5)$ \\
\hline Non-binary pronoun & $9(28.1 \%)$ & $4(9.5 \%)$ & $4(66.7 \%)$ & $9(13.4 \%)$ & $1(12.5 \%)$ & $12(18.2 \%)$ \\
\hline Identification with birth assigned sex & $9.0(22.5)$ & $10.0(18.8)$ & $21.0(31.8)$ & $9.0(18.5)$ & $0.0(2.0)$ & $12.0(21.0)$ \\
\hline Identification with opposite to birth assigned sex & $85.0(23.0)$ & $89.0(20.0)$ & $63.5(22.0)$ & $88.0(19.0)$ & $94.0(11.5)$ & $85.5(19.5)$ \\
\hline Real-life experience (always/often) & $15(46.9 \%)$ & $26(61.9 \%)$ & $2(33.3 \%)$ & $38(56.7 \%)$ & $7(87.5 \%)$ & $34(51.5 \%)$ \\
\hline Satisfaction with body appearance, median (IQR) & $20.0(23.5)$ & $15.0(29.0)$ & $15.0(15.2)$ & $19.0(28.2)$ & $20.0(14.0)$ & $15.0(27.0)$ \\
\hline Sexual preference: Androphilic & $3(9.4 \%)$ & $11(26.2 \%)$ & $1(16.7 \%)$ & $13(19.4 \%)$ & $1(12.5 \%)$ & $13(19.7 \%)$ \\
\hline Bisexual & $6(18.8 \%)$ & $8(19 \%)$ & $1(16.7 \%)$ & $12(17.9 \%)$ & $2(25 \%)$ & $12(18.2 \%)$ \\
\hline Gynophilic & $19(59.4 \%)$ & $20(47.6 \%)$ & $4(66.7 \%)$ & $35(52.2 \%)$ & $5(62.5 \%)$ & $34(51.5 \%)$ \\
\hline Asexual & $2(6.2 \%)$ & $0(0 \%)$ & $0(0 \%)$ & $2(3 \%)$ & $0(0 \%)$ & $2(3 \%)$ \\
\hline Unsure/Other & $1(3.1 \%)$ & $3(7.1 \%)$ & $0(0 \%)$ & $4(6 \%)$ & $0(0 \%)$ & $4(6.1 \%)$ \\
\hline Sexually active & $15(46.9 \%)$ & $21(50 \%)$ & $6(100 \%)$ & $30(44.8 \%)$ & $4(50 \%)$ & $32(48.5 \%)$ \\
\hline $\begin{array}{l}\text { Discrimination during past year because of gender identity } \\
\text { and expression, median (IQR) }\end{array}$ & $14.0(45.0)$ & $30.5(52.8)$ & $12.5(16.2)$ & $26.5(56.0)$ & $14.0(19.8)$ & $27.0(56.0)$ \\
\hline $\begin{array}{l}\text { Experienced lifetime trauma because of gender identity and } \\
\text { expression }\end{array}$ & $12(37.5 \%)$ & $20(47.6 \%)$ & $1(16.7 \%)$ & $31(46.3 \%)$ & $2(25 \%)$ & $30(45.5 \%)$ \\
\hline TCS Appearance Congruence, median (IQR) & $2.0(1.1)$ & $1.6(1.0)$ & $1.3(0.4)$ & $1.8(1.1)$ & $1.9(1.2)$ & $1.7(1.1)$ \\
\hline TCS Gender Identity Acceptance, median (IQR) & $4.0(1.0)$ & $4.3(2.0)$ & $3.5(0.6)$ & $4.0(2.0)$ & $4.7(0.5)$ & $4.0(2.0)$ \\
\hline
\end{tabular}

Cells show $n(\%)$ if not otherwise stated. Statistically significant factors $(p<0.05)$ derived by Pearson's chi-square test or Mann-Whitney $U$ test are written in boldface

$I Q R$ interquartile range, TCS Transgender Congruence Scale 
Table 4 Preferences for gender affirming treatments and associated factors for participants assigned female sex at birth

\begin{tabular}{|c|c|c|c|c|c|c|c|c|}
\hline \multirow[t]{2}{*}{ Assigned female } & \multicolumn{2}{|c|}{$\begin{array}{l}\text { Top surgery (mastec- } \\
\text { tomy) }\end{array}$} & \multicolumn{2}{|c|}{$\begin{array}{l}\text { External genital } \\
\text { surgery }\end{array}$} & \multicolumn{2}{|c|}{$\begin{array}{l}\text { Internal genital } \\
\text { surgery }\end{array}$} & \multicolumn{2}{|c|}{$\begin{array}{l}\text { Other surgery (e.g., } \\
\text { facial) }\end{array}$} \\
\hline & No, $n=6$ & Yes, $n=117$ & No, $n=41$ & Yes, $n=80$ & No $=41$ & Yes $=82$ & No, $n=99$ & Yes, $n=24$ \\
\hline Age, median (IQR) & $26.5(14.5)$ & $23.0(8.0)$ & $23.0(8.0)$ & $23.0(8.0)$ & $23.0(7.0)$ & $23.0(9.0)$ & $23.0(8.0)$ & $23.5(7.0)$ \\
\hline Partner status (single) & $4(66.7 \%)$ & $71(60.7 \%)$ & $26(63.4 \%)$ & $47(58.8 \%)$ & $27(65.9 \%)$ & $48(58.5 \%)$ & $63(63.6 \%)$ & $12(50 \%)$ \\
\hline Educational level $(<=12$ years $)$ & $5(83.3 \%)$ & $82(70.1 \%)$ & $27(65.9 \%)$ & $59(73.8 \%)$ & $27(65.9 \%)$ & $60(73.2 \%)$ & $70(70.7 \%)$ & $17(70.8 \%)$ \\
\hline $\begin{array}{l}\text { Working (not unemployed/sick-leave/ } \\
\text { other) }\end{array}$ & $1(16.7 \%)$ & $42(35.9 \%)$ & $17(41.5 \%)$ & $25(31.2 \%)$ & $17(41.5 \%)$ & $26(31.7 \%)$ & $35(35.4 \%)$ & $8(33.3 \%)$ \\
\hline Good social support & $4(66.7 \%)$ & $60(51.3 \%)$ & $19(46.3 \%)$ & $44(55 \%)$ & $14(34.1 \%)$ & $50(61 \%)$ & $53(53.5 \%)$ & $11(45.8 \%)$ \\
\hline Body mass index, median (IQR) & $28.0(6.7)$ & $23.4(8.4)$ & $23.7(7.4)$ & $23.3(8.9)$ & $23.9(6.7)$ & $23.4(9.4)$ & $23.3(8.7)$ & $24.8(7.5)$ \\
\hline Somatic morbidity & $2(33.3 \%)$ & $46(39.3 \%)$ & $21(51.2 \%)$ & $26(32.5 \%)$ & $17(41.5 \%)$ & $31(37.8 \%)$ & $40(40.4 \%)$ & $8(33.3 \%)$ \\
\hline Psychiatric morbidity & $6(100 \%)$ & $89(76.1 \%)$ & $30(73.2 \%)$ & $63(78.8 \%)$ & $30(73.2 \%)$ & $65(79.3 \%)$ & $74(74.7 \%)$ & $21(87.5 \%)$ \\
\hline $\begin{array}{l}\text { Any suicidal attempts, or past year } \\
\text { suicidal ideation or self-harm }\end{array}$ & $1(16.7 \%)$ & $74(63.2 \%)$ & $25(61 \%)$ & $49(61.3 \%)$ & $22(53.7 \%)$ & $53(64.6 \%)$ & $59(59.6 \%)$ & $16(66.7 \%)$ \\
\hline Perceived health, median (IQR) & $40.0(5.0)$ & $50.0(36.5)$ & $47.5(34.2)$ & $50.0(39.0)$ & $57.5(31.0)$ & $46.0(37.5)$ & $48.0(36.0)$ & $63.0(36.5)$ \\
\hline Debut age of gender incongruence & $14.5(8.2)$ & $13.0(8.0)$ & $13.0(8.0)$ & $12.0(9.0)$ & $14.0(5.0)$ & $11.0(9.8)$ & $12.0(8.0)$ & $13.0(9.5)$ \\
\hline Non-binary pronoun & $1(16.7 \%)$ & $10(8.5 \%)$ & $8(19.5 \%)$ & $3(3.8 \%)$ & $8(19.5 \%)$ & $3(3.7 \%)$ & $9(9.1 \%)$ & $2(8.3 \%)$ \\
\hline Identification with birth assigned sex & $16.0(45.0)$ & $0.0(7.0)$ & $3.0(13.0)$ & $0.0(4.2)$ & $2.0(11.0)$ & $0.0(5.8)$ & $0.0(7.8)$ & $2.0(8.5)$ \\
\hline $\begin{array}{l}\text { Identification with opposite to birth } \\
\text { assigned sex }\end{array}$ & $65.0(42.0)$ & $94.0(20.0)$ & $85.0(26.0)$ & $99.0(14.2)$ & $89.0(23.0)$ & $98.0(16.8)$ & $93.0(18.0)$ & $88.0(23.8)$ \\
\hline Real-life experience (always/often) & $5(83.3 \%)$ & $105(89.7 \%)$ & $36(87.8 \%)$ & $72(90 \%)$ & $38(92.7 \%)$ & $72(87.8 \%)$ & $92(92.9 \%)$ & $18(75 \%)$ \\
\hline $\begin{array}{l}\text { Satisfaction with body appearance, } \\
\text { median (IQR) }\end{array}$ & $44.0(16.0)$ & $10.0(22.0)$ & $16.0(19.0)$ & $10.0(22.2)$ & $21.0(18.2)$ & $7.0(20.0)$ & $10.0(24.5)$ & $16.0(16.2)$ \\
\hline Sexual preference: Androphilic & $1(16.7 \%)$ & $24(20.5 \%)$ & $12(29.3 \%)$ & $12(15 \%)$ & $10(24.4 \%)$ & $15(18.3 \%)$ & $20(20.2 \%)$ & $5(20.8 \%)$ \\
\hline Bisexual & $2(33.3 \%)$ & $13(11.1 \%)$ & $1(2.4 \%)$ & $13(16.2 \%)$ & $7(17.1 \%)$ & $8(9.8 \%)$ & $12(12.1 \%)$ & $3(12.5 \%)$ \\
\hline Gynophilic & $1(16.7 \%)$ & $41(35 \%)$ & $6(14.6 \%)$ & $36(45 \%)$ & $6(14.6 \%)$ & $36(43.9 \%)$ & $36(36.4 \%)$ & $6(25 \%)$ \\
\hline Asexual & $0(0 \%)$ & $18(15.4 \%)$ & $8(19.5 \%)$ & $10(12.5 \%)$ & $5(12.2 \%)$ & $13(15.9 \%)$ & $12(12.1 \%)$ & $6(25 \%)$ \\
\hline Unsure/Other & $2(33.3 \%)$ & $21(17.9 \%)$ & $14(34.1 \%)$ & $9(11.2 \%)$ & $13(31.7 \%)$ & $10(12.2 \%)$ & $19(19.2 \%)$ & $4(16.7 \%)$ \\
\hline Sexually active & $2(33.3 \%)$ & $58(49.6 \%)$ & $22(53.7 \%)$ & $37(46.2 \%)$ & $20(48.8 \%)$ & $40(48.8 \%)$ & $49(49.5 \%)$ & $11(45.8 \%)$ \\
\hline $\begin{array}{l}\text { Discrimination during past year } \\
\text { because of gender identity and } \\
\text { expression, median (IQR) }\end{array}$ & $35.0(30.0)$ & $46.0(48.8)$ & $49.5(43.8)$ & $38.0(54.8)$ & $55.0(40.5)$ & $41.0(54.2)$ & $41.0(54.0)$ & $45.0(40.2)$ \\
\hline $\begin{array}{l}\text { Experienced lifetime trauma because } \\
\text { of gender identity and expression }\end{array}$ & $2(33.3 \%)$ & $56(47.9 \%)$ & $20(48.8 \%)$ & $38(47.5 \%)$ & $19(46.3 \%)$ & $39(47.6 \%)$ & $50(50.5 \%)$ & $8(33.3 \%)$ \\
\hline $\begin{array}{l}\text { TCS Appearance Congruence, } \\
\text { median (IQR) }\end{array}$ & $3.1(0.8)$ & $1.7(0.8)$ & $1.7(0.8)$ & $1.7(0.8)$ & $1.8(0.8)$ & $1.7(0.7)$ & $1.7(0.9)$ & $1.6(0.8)$ \\
\hline $\begin{array}{l}\text { TCS Gender Identity Acceptance, } \\
\text { median (IQR) }\end{array}$ & $3.8(0.4)$ & $4.0(1.3)$ & $3.7(0.7)$ & $4.0(1.3)$ & $4.0(0.7)$ & $3.7(1.3)$ & $4.0(1.0)$ & $3.7(1.3)$ \\
\hline
\end{tabular}

Cells show $n(\%)$ if not otherwise stated. Statistically significant factors $(p<0.05)$ derived by Pearson's chi-square test or Mann-Whitney $U$ test are written in boldface

$I Q R$ interquartile range, TCS Transgender Congruence Scale

associated with the wish for internal genital surgery, such as preferring a binary pronoun (he) and reporting better social support.

\section{Multivariable Associations}

Table 5 shows the results from the logistic regression analyses aiming to investigate factors independently associated with preferences for gender affirming treatment across the entire cohort. Regarding the choice of predictor variables for internal genital surgery, we did not include sexual attraction pattern because of low numbers in the cross-tabulated cells. Birth assigned sex was a strong predictor in all models. Regarding a preference for genital surgery, associated variables were preferring a binary pronoun, having no somatic morbidity, and a lower debut age of gender incongruence. A wish for internal genital surgery (hysterectomy or salpingo-oophorectomy) was associated with having good social support. Stating a preference for other surgery (facial, chondrolaryngoplasty, vocal cord, or other) was associated with less often living openly according to gender identity. 
Table 5 Multivariable logistic regression-derived associations between background characteristics and preferences for gender affirming treatment among all participants

\begin{tabular}{|c|c|c|c|c|}
\hline & \multirow[t]{2}{*}{ B (SE) } & \multicolumn{3}{|c|}{ 95\% CI for odds ratio } \\
\hline & & Lower & Odds ratio & Upper \\
\hline \multicolumn{5}{|l|}{ Top surgery } \\
\hline Intercept & $3.11(1.08)$ & & & \\
\hline Birth assigned sex ( $\mathrm{aF}$ vs. aM) & $3.39(0.67)$ & 8.97 & 29.71 & 130.59 \\
\hline Age & $0.07(0.02)$ & 0.97 & 1.02 & 1.07 \\
\hline Support (partially/none vs. good) & $-1.01(0.55)$ & 0.11 & 0.36 & 1.02 \\
\hline Non-binary pronoun (vs. binary pronoun) & $-1.22(0.70)$ & 0.07 & 0.29 & 1.13 \\
\hline Identification with birth assigned sex & $-0.01(0.01)$ & 0.96 & 0.99 & 1.02 \\
\hline Satisfaction with body appearance & $0.01(0.02)$ & 0.97 & 1.01 & 1.04 \\
\hline TCS Appearance Congruence & $-1.37(0.46)$ & 0.10 & 0.25 & 0.63 \\
\hline \multicolumn{5}{|c|}{ Observations, $n=183$. Events, $n=157$. Pseudo- $R^{2}=0.30($ Cox-Snell $), 0.50$ (Nagelkerke). Model chi-square $(7)=64.55, p<0.05$} \\
\hline \multicolumn{5}{|c|}{ Genital surgery } \\
\hline Intercept & $2.67(1.52)$ & & & \\
\hline Birth assigned sex ( $\mathrm{aF}$ vs. aM) & $-2.26(0.68)$ & 0.02 & 0.10 & 0.36 \\
\hline Age & $0.04(0.03)$ & 0.99 & 1.04 & 1.12 \\
\hline Partner status (single vs. in a relationship) & $-0.09(0.59)$ & 0.28 & 0.92 & 2.95 \\
\hline Support (partially/none vs. good) & $-0.41(0.46)$ & 0.26 & 0.66 & 1.64 \\
\hline Somatic morbidity (Yes vs. No) & $-1.18(0.51)$ & 0.11 & 0.30 & 0.80 \\
\hline Suicide attempts, suicidal thoughts or self-harm (Yes vs. No) & $0.52(0.49)$ & 0.64 & 1.68 & 4.49 \\
\hline Debut age of gender incongruence & $-0.08(0.03)$ & 0.86 & 0.92 & 0.98 \\
\hline Non-binary pronoun (vs. binary pronoun) & $-1.93(0.77)$ & 0.03 & 0.14 & 0.62 \\
\hline Identification with birth assigned sex & $-0.01(0.01)$ & 0.96 & 0.99 & 1.03 \\
\hline \multicolumn{5}{|l|}{ Sexual attraction pattern: Androphilic (ref) ${ }^{\mathrm{a}}$} \\
\hline Asexual $^{\text {a }}$ & $-0.12(0.78)$ & 0.19 & 0.88 & 4.12 \\
\hline Bisexual $^{\text {a }}$ & $2.27(1.02)$ & 1.57 & 9.68 & 97.92 \\
\hline Gynophilic $^{\text {a }}$ & $1.23(0.60)$ & 1.07 & 3.42 & 11.46 \\
\hline Unsure/other ${ }^{\text {a }}$ & $-0.05(0.69)$ & 0.25 & 0.95 & 3.74 \\
\hline Sexually active (Yes vs. No) & $-0.79(0.61)$ & 0.13 & 0.45 & 1.47 \\
\hline TCS Appearance Congruence & $0.48(0.44)$ & 0.71 & 1.61 & 4.01 \\
\hline \multicolumn{5}{|c|}{ Observations, $n=169$. Events, $n=129$. Pseudo- $R^{2}=0.30$ (Cox-Snell), 0.44 (Nagelkerke). Model chi-square $(15)=57.90, p<0.05$} \\
\hline \multicolumn{5}{|c|}{ Internal genital surgery } \\
\hline Intercept & $2.35(1.74)$ & & & \\
\hline Age & $0.04(0.04)$ & 0.96 & 1.04 & 1.13 \\
\hline Support (partially/none vs. good) & $-0.97(0.47)$ & 0.15 & 0.38 & 0.93 \\
\hline Debut age of gender incongruence & $-0.10(0.04)$ & 0.83 & 0.91 & 0.98 \\
\hline Non-binary pronoun (vs. binary pronoun) & $-1.52(0.99)$ & 0.03 & 0.22 & 1.45 \\
\hline Identification with opposite to birth assigned sex & $0.00(0.01)$ & 0.97 & 1.00 & 1.02 \\
\hline Satisfaction with body appearance & $-0.03(0.01)$ & 0.94 & 0.97 & 0.99 \\
\hline \multicolumn{5}{|c|}{ Observations, $n=122$. Events, $n=82$. Pseudo- $R^{2}=0.22$ (Cox-Snell), 0.30 (Nagelkerke). Model chi-square $(6)=30.08, p<0.05$} \\
\hline \multicolumn{5}{|c|}{ Other surgery } \\
\hline Intercept & $2.96(1.14)$ & & & \\
\hline Birth assigned sex ( $\mathrm{aF}$ vs. $\mathrm{aM})$ & $-3.38(0.50)$ & 0.01 & 0.03 & 0.09 \\
\hline Age & $0.01(0.02)$ & 0.97 & 1.01 & 1.06 \\
\hline Support (partially/none vs. good) & $0.25(0.45)$ & 0.53 & 1.28 & 3.14 \\
\hline Non-binary pronoun (vs. binary pronoun) & $0.12(0.71)$ & 0.27 & 1.13 & 4.43 \\
\hline Identification with birth assigned sex & $0.01(0.02)$ & 0.98 & 1.01 & 1.04 \\
\hline Real-life experience (always/often vs. sometimes/never) & $-1.28(0.57)$ & 0.09 & 0.28 & 0.85 \\
\hline TCS Appearance Congruence & $-0.25(0.34)$ & 0.39 & 0.78 & 1.47 \\
\hline
\end{tabular}

Significant parameters' odds ratios and $95 \%$ confidence intervals (95\% CIs) in boldface

$a F$ assigned female, $a M$ assigned male, TCS Transgender Congruence Scale

${ }^{a}$ Wald test showing non-significant result for the attraction pattern variable overall (chi-square $(4)=9.3, p>0.05$ ) 


\section{Discussion}

In this sample of transgender people seeking gender dysphoria health services in Sweden 2016-2019, almost half the sample did not want "full" surgical treatment (top surgery and genital surgery) but had specific preferences. Nearly everyone wanted cross-sex hormonal therapy and some sort of gender affirming surgery. Several factors were associated with a preference for gender affirming surgery in the group that had not yet received such treatment. For example, identification with a binary pronoun was associated with a wish for genital surgery.

Few studies have explored the wishes and motives regarding gender affirming treatment in recent years. In a Dutch study from 2015, the majority of gender dysphoric patients wanted "full" treatment, including hormones, top surgery, and genital surgery. Among those who wanted partial gender affirming treatment, outwardly visible treatments such as hormonal therapy or chest surgery were more commonly desired than genital surgery (Beek et al., 2015). Overall, the relative proportions of aF and aM individuals in the current study that wanted top surgery and genital surgery correspond to prior Dutch findings (Beek et al., 2015; Hage \& Karim, 2000). In contrast, the number of people receiving gender affirming surgery in the USA is lower, around 13-35\% (Kailas et al., 2017; Lane et al., 2018). This might reflect differences in the countries' social insurance systems. The incidence of gender affirming genital surgery in the USA is rising as more people have insurance coverage (Canner et al., 2018).

Preferring a non-binary pronoun was associated with being less interested in genital surgery, corresponding to previous reports (Beek et al., 2015; Jacobsson et al., 2017). A non-binary gender identity entails many different individual definitions and experiences, all rejecting the gender binary. In our sample of people in contact with health care, $11.2 \%$ did not identify with a binary pronoun. It has previously been estimated that about a third of transgender people have a non-binary gender identity, but are less likely to seek gender affirming health care (Matsuno \& Budge, 2017). It is important to note that this study focuses on clinically referred participants, meaning that we did not aim to describe preferences of all transgender people in Sweden. Having a high interest in the various types of gender affirming treatment is likely a main reason for many transgender people to seek healthcare. For illustration, $0.5 \%$ of the general population in Stockholm County, Sweden, declared at least some interest in hormonal or surgical gender affirming treatment, in a survey study by Åhs et al. (Åhs et al., 2018). Sixteen percent of the people declaring to identify with another than their birth assigned sex wished to have gender-affirming treatment in the same survey, suggesting that among transgender people not seeking health care, preferences for treatment are lower than in our findings.

As expected, there were several differences in treatment preferences between the $\mathrm{aM}$ and $\mathrm{aF}$ groups, e.g., regarding top surgery. Feminizing hormonal treatment may cause satisfying breast growth (Coleman et al., 2012), and masculinizing chest surgery is widely observed to be of high priority for many aF individuals early in their social transition to facilitate being perceived publicly according to gender identity and to alleviate body dysphoria (van de Grift et al., 2017). Fewer aF than aM were certain that they wanted external genital surgery, consistent with expectations of surgical complications or unsatisfactory results being a common motive for not wanting surgical treatment (Beek et al., 2015) and that complication and revision rates for genital surgery are higher for masculinizing procedures (Pan \& Honig, 2018). According to a Swedish pilot survey study with 47 transgender men, the top priorities prior to genital surgery were preserved tactile sensation and orgasm capability (Jacobsson et al., 2017). In the aF group, the wish for hysterectomy or salpingo-oophorectomy was reported by two-thirds of participants. These abdominal surgical procedures, which can be performed laparoscopically with low complication rates (Weyers et al., 2010), eliminate the need for yearly ultrasound examinations as well as the risk for ovarian or cervical cancer (Weyers et al., 2010) but are not outwardly visible. At present in Sweden, feminizing facial surgery is not covered by the public health insurance except for exceptional cases (The National Board of Health \& Welfare, 2015), although in our results, this kind of surgery was desired by the majority of aM persons. Transgender women may feel they do not "pass" as females despite adjusting their body language, voice, and undertaking the other kinds of gender affirming treatment, since feminizing hormones do not change the masculine bone structure.

Two factors not directly related to gender dysphoria appear to play a role in the preferences for gender affirming treatments, somatic morbidities, and social support. Participants with reported somatic morbidity were $70 \%$ less likely to report a wish for genital surgery. The reasons behind this association as well as whether there are specific somatic conditions behind this finding, deserve further investigation. On the other hand, good social support was associated with wish for internal genital surgery among aF. Social support is a known good prognostic factor for people with gender dysphoria (Köhler et al., 2019), but its influence in the preferences of gender affirming treatment needs to be further explored.

This study has several limitations. Approximately $30 \%$ of the people who were asked to participate declined, and additional people were excluded since they did not fill in 
basic demographic data. Differential recruitment rates imply that the northern parts of Sweden may be underrepresented. Still, a main strength of this study is the recruitment of participants from all Swedish gender dysphoria centers. As in previous epidemiological studies, the sample mainly consisted of young adults (Arcelus et al., 2015; Dhejne et al., 2014), why the results might not be applicable for children or adolescents below age 15 nor older people. Recruitment occurred early in the assessment process, and many had not yet received a formal diagnosis of Gender Dysphoria or Transsexualism. However, usually, the vast majority of adults seeking treatment for gender dysphoria in Sweden receive the diagnosis. Of note, this study presents multiple exploratory association tests without correction for multiple testing, and we invite the reader to interpret the results in light of the totality of tests performed. Also, while the sample size is comparable to similar studies in this field (Claes et al., 2015; Factor \& Rothblum, 2008; Heylens et al., 2014), results should preferably be replicated in a larger sample since certain combinations of variables were rare, affecting statistical power especially in the multivariable analyses. Aggregating the data across gender categories on treatment types like top surgery and genital surgery in the multivariable analyses was a necessary measure considering sample size, but suboptimal for interpretation. Moreover, all data in this study were self-reported and most questions examined were not part of validated questionnaires. Several questions on subjective experiences were designed with continuous scales to increase validity, while for some characteristics, e.g., gender identity, questions applied pre-defined categorical answers for practical reasons. The use of continuous scales or validated questionnaires may shed further light on the associations in this study. This cross-sectional sub-study only reflects the desires at one point during the evaluation process, whereas a recently published cross-sectional study of a German convenience sample of 415 transgender persons implies that needs and wishes regarding decision-making and supportive interventions may fluctuate during the treatment process (Mayer et al., 2019).

Findings of a Swedish qualitative study highlight the importance of health care professionals' attitudes and knowledge for the wellbeing of the patient during the gender affirming treatment process (von Vogelsang et al., 2016). In a cross-sectional survey on a self-selected sample of 796 Swedish transgender people, negative health care experiences were associated with worse self-rated health, disability, and quality of life (Zeluf et al., 2016). For a better understanding of the needs of this population and for an efficient planning of the allocation of health care resources, knowledge about requests for treatment and influencing factors are imperative. Future studies may advance the knowledge on treatment preferences by exploring underlying motivations, concerns, and priorities. Desire for gender affirming treatment among transgender people not currently seeking health care also needs to be further examined. Moreover, researchers may investigate if desires stated in the beginning of the assessment process fluctuate or change during the time of evaluation and commencing treatment.

\section{Conclusion}

Gender affirming treatment preferences were shown to vary among transgender people seeking health care for gender dysphoria in Sweden, implying a need for flexible treatment options. The role of somatic comorbidities should also be further explored, and support should be given to transgender people who lack social support and do not live openly according to their gender identity, as these factors may influence their preferences for treatment. Finally, our findings point to an unmet need for facial surgery with a majority of $\mathrm{aM}$ expressing a desire for this kind of surgery.

Supplementary Information The online version contains supplementary material available at https://doi.org/10.1007/s13178-021-00650-2.

Funding Open access funding provided by Uppsala University.

Open Access This article is licensed under a Creative Commons Attribution 4.0 International License, which permits use, sharing, adaptation, distribution and reproduction in any medium or format, as long as you give appropriate credit to the original author(s) and the source, provide a link to the Creative Commons licence, and indicate if changes were made. The images or other third party material in this article are included in the article's Creative Commons licence, unless indicated otherwise in a credit line to the material. If material is not included in the article's Creative Commons licence and your intended use is not permitted by statutory regulation or exceeds the permitted use, you will need to obtain permission directly from the copyright holder. To view a copy of this licence, visit http://creativecommons.org/licenses/by/4.0/.

\section{References}

Åhs, J. W., Dhejne, C., Magnusson, C., Dal, H., Lundin, A., Arver, S., \& Kosidou, K. (2018). Proportion of adults in the general population of Stockholm County who want gender-affirming medical treatment. PLoS One, 13(10), e0204606. https://doi.org/10.1371/ journal.pone.0204606

American Psychiatric Association. (2013). Diagnostic and statistical manual of mental disorders (5th ed.): Arlington.

Arcelus, J., Bouman, W. P., Van Den Noortgate, W., Claes, L., Witcomb, G., \& Fernandez-Aranda, F. (2015). Systematic review and meta-analysis of prevalence studies in transsexualism. European Psychiatry, 30(6), 807-815. https://doi.org/10. 1016/j.eurpsy.2015.04.005

Beek, T. F., Kreukels, B. P., Cohen-Kettenis, P. T., \& Steensma, T. D. (2015). Partial treatment requests and underlying motives of applicants for gender affirming interventions. The Journal of Sexual Medicine, 12(11), 2201-2205. https://doi.org/10.1111/jsm.13033

Canner, J. K., Harfouch, O., Kodadek, L. M., Pelaez, D., Coon, D., Offodile, A. C. 2nd., \& Lau, B. D. (2018). Temporal trends in 
gender-affirming surgery among transgender patients in the United States. JAMA Surgery, 153(7), 609-616. https://doi.org/10.1001/ jamasurg.2017.6231

Claes, L., Bouman, W. P., Witcomb, G., Thurston, M., FernandezAranda, F., \& Arcelus, J. (2015). Non-suicidal self-injury in trans people: Associations with psychological symptoms, victimization, interpersonal functioning, and perceived social support. The Journal of Sexual Medicine, 12(1), 168-179. https://doi.org/10. 1111 jsm. 12711

Cohen-Kettenis, P. T., \& Pfäfflin, F. (2010). The DSM diagnostic criteria for gender identity disorder in adolescents and adults. Archives of Sexual Behavior, 39(2), 499-513. https://doi.org/10. 1007/s10508-009-9562-y

Coleman, E., Bockting, W., Botzer, M., Cohen-Kettenis, P., Cuypere, G., Feldman, J., \& Zucker, K. (2012). Standards of care for the health of transsexual, transgender, and gender-nonconforming people, Version 7. International Journal of Transgenderism, 13, 165-232. https://doi.org/10.1080/15532739.2011.700873

Dhejne, C., Öberg, K., Arver, S., \& Landén, M. (2014). An analysis of all applications for sex reassignment surgery in Sweden, 1960-2010: Prevalence, incidence, and regrets. Archives of Sexual Behavior, 43(8), 1535-1545. https://doi.org/10.1007/s10508-014-0300-8

Dhejne, C., Van Vlerken, R., Heylens, G., \& Arcelus, J. (2016). Mental health and gender dysphoria: A review of the literature. International Review of Psychiatry, 28(1), 44-57. https://doi.org/10.3109/ 09540261.2015.1115753

Factor, R., \& Rothblum, E. (2008). Exploring gender identity and community among three groups of transgender individuals in the United States: MTFs, FTMs, and genderqueers. Health Sociology Review, 17(3), 235-253. https://doi.org/10.5172/hesr.451. 17.3.235

Hage, J. J., \& Karim, R. B. (2000). Ought GIDNOS get nought? Treatment options for nontranssexual gender dysphoria. Plastic and Reconstructive Surgery, 105(3), 1222-1227. https://doi.org/10. 1097/00006534-200003000-00063

Heylens, G., Elaut, E., Kreukels, B. P., Paap, M. C., Cerwenka, S., Richter-Appelt, H., \& De Cuypere, G. (2014). Psychiatric characteristics in transsexual individuals: Multicentre study in four European countries. British Journal of Psychiatry, 204(2), 151-156. https://doi.org/10.1192/bjp.bp.112.121954

Jacobsson, J., Andréasson, M., Kölby, L., Elander, A., \& Selvaggi, G. (2017). Patients' priorities regarding female-to-male gender affirmation surgery of the genitalia-A pilot study of 47 patients in Sweden. The Journal of Sexual Medicine, 14(6), 857-864. https:// doi.org/10.1016/j.jsxm.2017.04.005

Johansson, A., Sundbom, E., Höjerback, T., \& Bodlund, O. (2010). A five-year follow-up study of Swedish adults with gender identity disorder. Archives of Sexual Behavior, 39(6), 1429-1437. https:// doi.org/10.1007/s10508-009-9551-1

Kailas, M., Lu, H. M. S., Rothman, E. F., \& Safer, J. D. (2017). Prevalence and types of gender-affirming surgery among a sample of transgender endocrinology patients prior to state expansion of insurance coverage. Endocrine Practice, 23(7), 780-786. https:// doi.org/10.4158/ep161727.or

Köhler, A., Becker, I., Richter-Appelt, H., Cerwenka, S., Kreukels, B., van de Grift, T., \& Nieder, T. O. (2019). Treatment experiences and social support in individuals with gender incongruence/gender dysphoria - A ENIGI 5 year follow-up study in three European countries. Psychotherapie, Psychosomatik, Medizinische Psychologie, 69(8), 339-347. https://doi.org/10.1055/a-0806-6892

Kozee, H. B., Tylka, T. L., \& Bauerband, L. A. (2012). Measuring transgender individuals' comfort with gender identity and appearance: Development and validation of the transgender congruence scale. Psychology of Women Quarterly, 36(2), 179-196. https:// doi.org/10.1177/0361684312442161
Lane, M., Ives, G. C., Sluiter, E. C., Waljee, J. F., Yao, T. H., Hu, H. M., \& Kuzon, W. M. (2018). Trends in gender-affirming surgery in insured patients in the United States. Plastic and Reconstructive Surgery. Global Open, 6(4), e1738. https://doi.org/10.1097/gox. 0000000000001738

Matsuno, E., \& Budge, S. L. (2017). Non-binary/genderqueer identities: A critical review of the literature. Current Sexual Health Reports, 9(3), 116-120. https://doi.org/10.1007/s11930-017-0111-8

Mayer, T. K., Koehler, A., Eyssel, J., \& Nieder, T. O. (2019). How gender identity and treatment progress impact decision-making, psychotherapy and aftercare desires of trans persons. Journal of Clinical Medicine, 8(5). https://doi.org/10.3390/jcm8050749

Ministry of Social Affairs. (1972). Svensk författningssamling 1972:119. The Act on the determination of gender in certain cases. Retrieved from Stockholm: https://www.riksdagen.se/ sv/dokument-lagar/dokument/svensk-forfattningssamling/lag1972119-om-faststallande-av_sfs-1972-119

Murad, M. H., Elamin, M. B., Garcia, M. Z., Mullan, R. J., Murad, A., Erwin, P. J., \& Montori, V. M. (2010). Hormonal therapy and sex reassignment: A systematic review and meta-analysis of quality of life and psychosocial outcomes. Clinical Endocrinology - Oxford, 72(2), 214-231. https://doi.org/10.1111/j.1365-2265. 2009.03625.x

Pan, S., \& Honig, S. C. (2018). Gender-affirming surgery: Current concepts. Current Urology Reports, 19(8), 62. https://doi.org/10. 1007/s11934-018-0809-9

Richards, C., Bouman, W. P., Seal, L., Barker, M. J., Nieder, T. O., \& T'Sjoen, G. (2016). Non-binary or genderqueer genders. International Review of Psychiatry, 28(1), 95-102. https://doi.org/10. 3109/09540261.2015.1106446

Södersten, M., Johansson, A., Beckman, U., Östberg, P., \& Dhejne, C. (2016). Swedish translation of the Transgender Congruence Scale (TCS) according to WHO:s guidelines.

Statistics Sweden (SCB). (2019). Population statistics by county, civil status, age and sex. Years 1968-2018. Retrieved from Stockholm: www.scb.se

The National Board of Health and Welfare. (2015). Care of adults with gender dysphoria - National recommendations. Retrieved from https://www.socialstyrelsen.se/globalassets/sharepoint-dokument/ artikelkatalog/kunskapsstod/2015-4-7.pdf

The National Board of Health and Welfare. (2019). Errands statistics of the legal advisory board of the National Board of Health and Welfare 2004-2018. Retrieved from Stockholm: Ministry of Social Affairs.

van de Grift, T. C., Elaut, E., Cerwenka, S. C., Cohen-Kettenis, P. T., $\&$ Kreukels, B. P. C. (2018). Surgical satisfaction, quality of life, and their association after gender-affirming surgery: A followup study. Journal of Sex and Marital Therapy, 44(2), 138-148. https://doi.org/10.1080/0092623x.2017.1326190

van de Grift, T. C., Elfering, L., Bouman, M. B., Buncamper, M. E., \& Mullender, M. G. (2017). Surgical indications and outcomes of mastectomy in transmen: A prospective study of technical and self-reported measures. Plastic and Reconstructive Surgery, 140(3), 415e-424e. https://doi.org/10.1097/prs. 0000000000003607

von Vogelsang, A. C., Milton, C., Ericsson, I., \& Strömberg, L. (2016). 'Wouldn't it be easier if you continued to be a guy?' - A qualitative interview study of transsexual persons' experiences of encounters with healthcare professionals. Journal of Clinical Nursing, 25(23-24), 3577-3588. https://doi.org/10.1111/jocn.13271

Weyers, S., De Sutter, P., Hoebeke, S., Monstrey, G., \& G, T. S., Verstraelen, H., \& Gerris, J. (2010). Gynaecological aspects of the treatment and follow-up of transsexual men and women. Facts Views vis Obgyn, 2(1), 35-54. 
Winter, S., Diamond, M., Green, J., Karasic, D., Reed, T., Whittle, S., \& Wylie, K. (2016). Transgender people: Health at the margins of society. Lancet, 388(10042), 390-400. https://doi.org/10.1016/ s0140-6736(16)00683-8

Zeluf, G., Dhejne, C., Orre, C., Nilunger Mannheimer, L., Deogan, C., Höijer, J., \& Ekéus Thorson, A. (2016). Health, disability and quality of life among trans people in Sweden-A web-based survey. BMC Public Health, 16(1), 903. https://doi.org/10.1186/ s12889-016-3560-5

Publisher's Note Springer Nature remains neutral with regard to jurisdictional claims in published maps and institutional affiliations. 